\title{
Electromechanics of fluidized beds of nanoparticles
}

\author{
M. J. Espin, ${ }^{1}$ J. M. Valverde, ${ }^{2}$ M. A. S. Quintanilla, ${ }^{2}$ and A. Castellanos ${ }^{2}$ \\ ${ }^{1}$ Department of Applied Physics II, University of Seville, Avenida Reina Mercedes, 41012 Seville, Spain \\ ${ }^{2}$ Department of Electronics and Electromagnetism, University of Seville, Avenida Reina Mercedes, 41012 Seville, Spain
}

(Received 9 May 2008; revised manuscript received 16 December 2008; published 27 January 2009)

\begin{abstract}
The electromechanical behavior of a gas-fluidized bed of insulating silica nanoparticles is investigated. When fluidized by gas, these nanoparticles form highly porous agglomerates of size of the order of hundreds of microns, which gives rise to a nonbubbling fluidization regime. Bed expansion is enhanced by an imposed alternating electric field for oscillation frequencies in the range between tens and hundreds of hertzs and field strengths of about $1 \mathrm{kV} / \mathrm{cm}$. Nanoparticle agglomerates are naturally charged and experience forced oscillations that cause an increase of the gas flow shear on their surface. As a consequence, the agglomerate size is expected to decrease, which can explain the observed behavior. A model based on the balance between attractive and flow shear forces is presented that accounts for agglomerate size reduction as the strength of the field is increased.
\end{abstract}

DOI: 10.1103/PhysRevE.79.011304

PACS number(s): 45.70.-n, 47.55.Lm, 81.07.Wx, 84.30.Vn

\section{INTRODUCTION}

Fundamental problems in our modern world, such as reducing air pollution from diesel exhaust and filtration of smoke emissions from asphaltic pavement recycling process, motivated Melcher to develop electrofluidized beds for collection of submicron particles [1]. In a fluidized bed the granular material rests on a porous plate through which gas is distributed. At a certain gas flow the drag force balances the material weight per unit area and the grains become fluidized. Melcher stressed a fluidized bed by imposing an electric field that effectively polarized the grains, which thus acted as collection sites for the charged pollutants entrained in the fluidizing gas, serving the function of electrostatic precipitators. In Melcher's investigations, semiinsulating wet grains were used to allow for charge exchange during collisions, thus preventing charge accumulation on the grains. Tardos et al. [2] investigated the efficiency of contaminant collection in a dry fluidized bed due to the charges generated naturally on insulating polystyrene spheres. The dry fluidized bed demonstrated very high cleaning efficiencies, even without an externally imposed electric field, due to the strong charge buildup on the granules [2]. Elsdon and Shearer [3] reported that the application of an ac electric field to a dry bubbling bed of $0.24 \mathrm{~mm}$ polymer particles caused an appreciable increase of the net transport of heat for field oscillation frequencies of about $100 \mathrm{~Hz}$. This behavior could be explained semiquantitatively in terms of the enhanced agitation of the particles, which were charged naturally by contact electrification and, thus, experienced forced oscillations around their mean position. However, a major concern that besets fluidized bed filters lies in the bypassing of the contaminants through large gas bubbles that usually develop in gas-fluidized beds of coarse grains (grain size $d_{p} \geq 100 \mu \mathrm{m}$ ) [4]. In contrast, it has been shown that gas-fluidized beds of some micron and submicron particles exhibit nonbubbling fluidlike behavior $[5,6]$. For example, powders of moderate density nanoparticles (such as silica) can be uniformly fluidized by gas $[5,7]$. Fluidization of nanoparticles, providing extremely high specific surface area, has become thus one of the most promising techniques for emerging industrial applications. The use of fluidized beds of titania nanoparticles has been proposed to remove pollutant nitrogen oxides from air by photocatalytic reaction [8]. Fluidization of $\mathrm{Co} / \mathrm{Al}_{2} \mathrm{O}_{3}$ nanoparticles has been employed to improve the catalytic activity of reforming methane with $\mathrm{CO}_{2}$ [9]. Fluidized beds of silica nanoparticles coated with catalysts have been thought to be an alternative to bubbling beds used in the oil industry [10] among other potential uses. In this paper we investigate the electromechanical behavior of a dry fluidized bed of silica nanoparticles stressed by a cross-flow ac electric field.

\section{EXPERIMENTAL SETUP AND PROCEDURE}

The fluidized bed apparatus consists of a vertical $2.54 \mathrm{~cm}$ internal diameter polycarbonate vessel, $16.2 \mathrm{~cm}$ in height, with a sintered stainless steel plate at the bottom that acts as a gas distributor. The fluidization cell is placed between two parallel electrodes $8 \mathrm{~cm}$ width apart. One of the electrodes is grounded and alternating high voltage $V$ is applied to the other from an oscillator and amplifier network. According to three-dimensional (3D) numerical calculations the field strength within the bed is approximately uniform. A cross section with the electric field and equipotential lines obtained from the numerical calculations are shown in Fig. 1. As can be seen, the field inside the vessel is quite homogeneous with a slightly smaller strength than outside the fluidization vessel due to wall effects. The calculations performed in the horizontal plane at different levels show that the disturbance created by the metallic distributor is important only in the very close vicinity of the distributor, and that at the level of the lower edge of the electrodes this perturbation is not significant.

The power supply system allowed us to provide peak field strengths up to $E_{0} \simeq 2 \mathrm{kV} / \mathrm{cm}$ over a frequency range up to $10 \mathrm{kHz}$. Different types of wave form, sinusoidal, square and triangular shape, were applied. The gas flow (dry air) to the cell is controlled by a MKS Instruments mass flow controller with a full range from 0 to $2000 \mathrm{~cm}^{3} / \mathrm{min}$. The nanopowder 


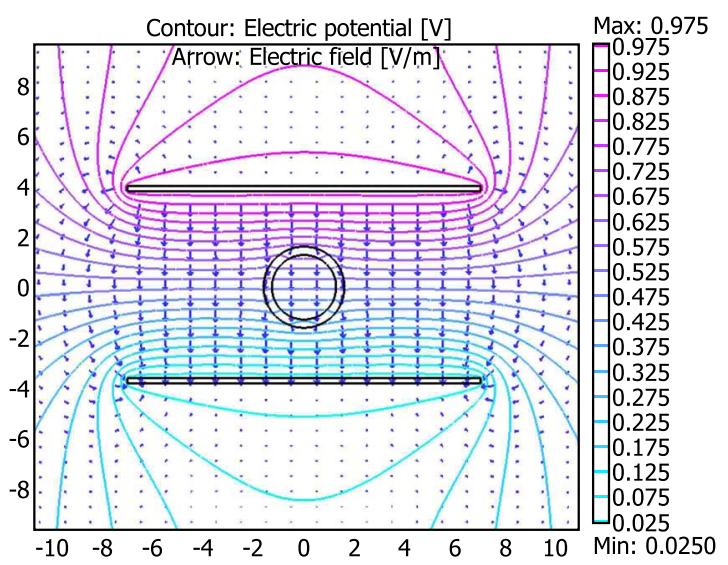

FIG. 1. (Color online) Cross section of the experimental system showing the electric field and equipotential lines obtained from numerical calculations. The electrodes and the cell have been drawn to scale, with the distances in $\mathrm{cm}$. One electrode was set to voltage 1 and the other one was grounded.

tested is Aerosil R974, which consists of hydrophobic silica (particle density $\rho_{p}=2250 \mathrm{~kg} / \mathrm{m}^{3}$ and particle size $d_{p}$ $=12 \mathrm{~nm}$ ). The nanopowder is presieved (sieve opening of $425 \mu \mathrm{m})$, and initialized in the fluidization cell by applying a sufficiently high gas flow. Once the initialized bed reaches a uniform state, the gas flow is set to a given value and the bed height in the stationary state is measured. Then the electric field is turned on and the stationary bed height after application of the field is measured. Additionally, scanning electron microscopy of the nanopowder has been performed.

\section{EXPERIMENTAL RESULTS AND DISCUSSION}

\section{A. Bed expansion in the absence of applied electric field}

In Fig. 2 we have plotted experimental data of the particle volume fraction $\phi_{0}$ as a function of the superficial gas velocity $v_{g}$ without electric field applied. Note that small values of $v_{g}$ are required for uniform fluidization $\left(v_{g} \sim 1 \mathrm{~cm} / \mathrm{s}\right)$ as compared to values typically required for fluidization of bubbling beds of coarse grains $\left(v_{g} \sim 1 \mathrm{~m} / \mathrm{s}\right)$ [2]. Figure 2 shows the different fluidization regimes displayed by the nanofluidized bed. For $v_{g} \gtrsim 1 \mathrm{~cm} / \mathrm{s}$ the nanofluidized bed shows a fluidlike behavior and nanoparticle agglomerates are seen to display a pseudoturbulent random motion. In this uniform fluidlike regime, macroscopic bubbles are not visible, and the gas pressure adjusts to the powder weight per unit area. The height of the free surface and the mass of elutriated powder increase monotonically as the gas velocity is increased and we observe a full suppression of the bubbling regime, i.e., the fluidized bed transits gradually to elutriation. Since bed expansion is not curtailed by the development of macroscopic gas bubbles, the bed is seen to expand uniformly as $v_{g}$ is increased. Nanoparticle agglomerates elutriate off the bed appreciably when the gas velocity is further increased above $v_{g} \simeq 2.7 \mathrm{~cm} / \mathrm{s}$. For $v_{g} \lesssim 1 \mathrm{~cm} / \mathrm{s}$, free surface instabilities are observed due to the formation of transient solid networks that are increasingly predominant as the gas velocity is further decreased. This is the transitional regime.

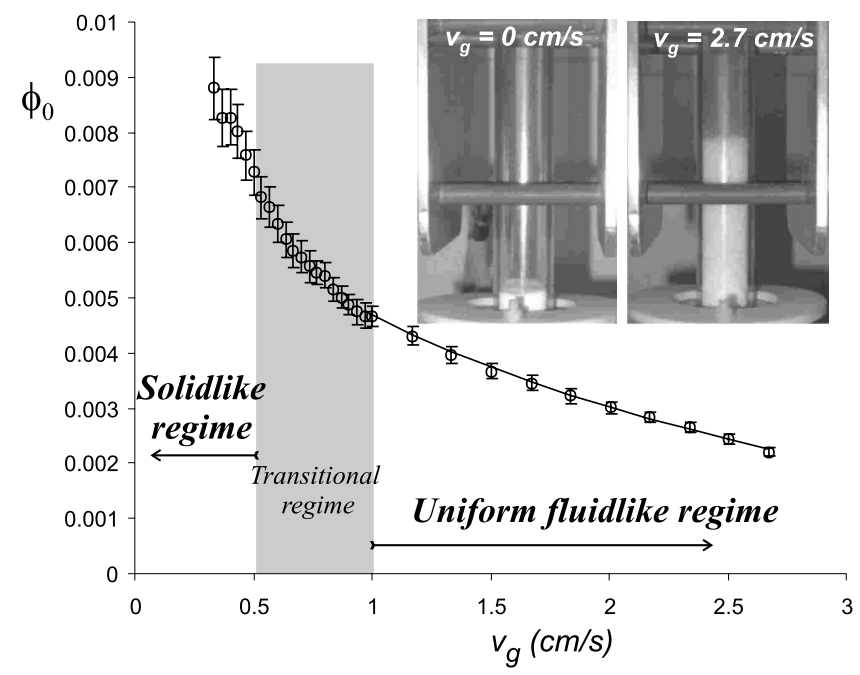

FIG. 2. Particle volume fraction $\phi_{0}$ vs superficial gas velocity $v_{g}$ without electric field applied. Fluidization regimes have been delineated. The solid line represents the modified RZ equation fit to the data in the fluidlike regime. Photographs in the inset illustrate bed expansion.

For $v_{g} \leqq 0.6 \mathrm{~cm} / \mathrm{s}$, the solidlike structure is just perturbed by little volcanoes that are visible at the free surface and for $v_{g} \lesssim 0.5 \mathrm{~cm} / \mathrm{s}$, the nanofluidized bed is fully jammed. This is the solidlike regime. In the solidlike regime the bed retains a small, but nonvanishing, yield strength due to the enduring adhesive contacts established between the jammed agglomerates. Note that the particle volume fraction in the jammed state is rather low, which is attributable to the extremely high porosity of the nanoparticle agglomerates (about 99\% of the agglomerate volume is free of particles [7]). The existence of these fluidization regimes has been previously reported [11] and can be inferred either from bed expansion observations or measurements of the mechanical strength of the fluidized bed and local reflectance (see Ref. [11] for further details).

According to the scanning electron microscopy analysis by Yao et al. [12] on 7-16 nm silica particles, the primary nanoparticles form multistage agglomerates by three steps. First, primary nanoparticles are seen to be agglomerated into 3D netlike structures (let us call them subagglomerates). Second, the subagglomerates are agglomerated into simple agglomerates of size $d_{S}$ of the order of tens of microns and existing before fluidization. Scanning electron microscope photographs performed by us of simple agglomerates of Aerosil R974 are shown in Fig. 3, where it is seen that their size fits in this typical size. When the nanopowder is fluidized, the pre-existing simple agglomerates further agglomerate into so-called complex agglomerates of size $d_{C}$ of the order of hundreds of microns. The suppression of gas bubbles in nanofluidized beds is causally related to the formation of these highly porous multistage agglomerates [12] The hydrodynamic and gyration radius of fine particle agglomerates are similar since they screen the gas flow quite efficiently [13]. Thus, a phenomenological interpretation of the expanded uniform state of nanofluidized beds carried out in previous works has been to consider complex agglomerates as effective low-density spheres, which may exhibit nonbubbling fluidization [11]. 

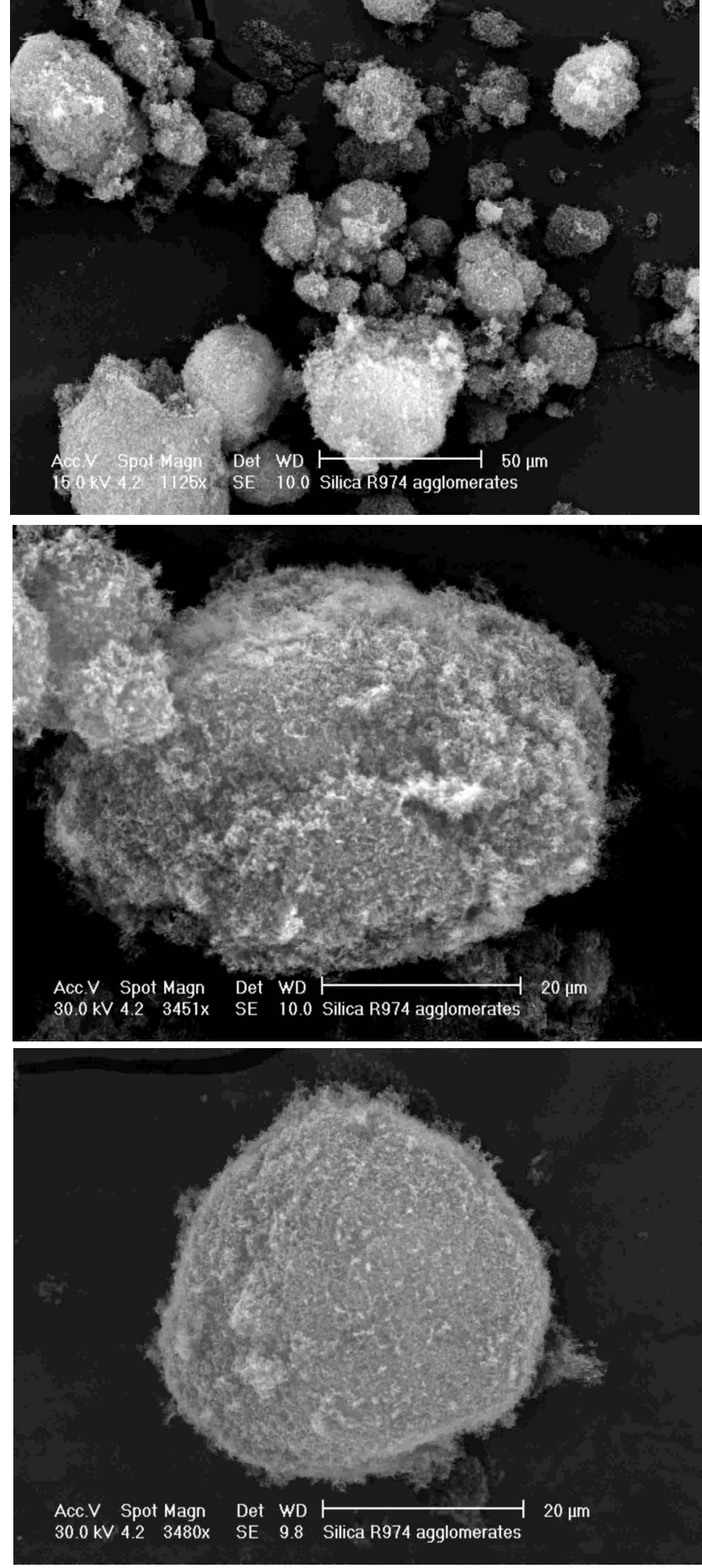

FIG. 3. SEM photographs of simple agglomerates of the nanopowder used in the experiments (Aerosil R974).

The Richardson-Zaki (hereafter termed as RZ) empirical equation [14]

$$
\frac{v_{g}}{v_{p}}=(1-\phi)^{n}
$$

relates the particle volume fraction $\phi$ of the fluidized bed to the superficial gas velocity $v_{g}$ (or the settling velocity in sedimentation). Here $v_{p}$ is the sedimentation velocity of a single particle, $v_{p}=(1 / 18)\left(\rho_{p}-\rho_{g}\right) g d_{p}^{2} / \mu$ in the laminar regime, where $\rho_{p}$ is the particle density, $\rho_{g}$ is the gas density, $g$ is the gravity field, $d_{p}$ is the particle size, $\mu$ is the viscosity of the gas and $n$ is a parameter of order 5 in the small Reynolds number limit. Theoretical considerations by Batchelor [15] leaded to $v_{g} / v_{p} \simeq 1-5.6 \phi$ for very diluted suspensions of non-Brownian hard spheres in the limit of small Reynolds number. Thus, the sedimentation velocity predicted by Eq. (1) conforms with the theoretical dilute limit by Batchelor for $n=5.6$. The RZ equation was originally intended to describe the expansion of liquid-fluidized beds [14], where the fluidized particles were not agglomerated, and fluidization was usually uniform. Thus, Eq. (1) is not suitable for fluidized beds of fine particles, which are usually agglomerated. The simplest approximation to account for agglomeration in fluidized beds of nanoparticles is to assume an ideal system of monodisperse complex agglomerates with a hydrodynamic radius equal to their radius of gyration due to the effective screening of the gas flow by the agglomerates [13]. Then the Richardson-Zaki law would be

$$
\frac{v_{g}}{v_{C}}=\left(1-\phi_{C}\right)^{n},
$$

where $v_{C}$ is the settling velocity of an individual complex agglomerate and $\phi_{C}$ is the volume fraction occupied by the complex agglomerates. This is the so-called modified Richardson-Zaki (hereafter termed as mRZ) equation. The extension of the RZ equation for fluidized systems of agglomerated fine particles was first reported in Ref. [16].

The settling velocity of an individual complex agglomerate can be calculated from the balance of the weight of the complex agglomerate, $W_{C}=N(1 / 6) \pi d_{p}^{3} \rho_{p} g$, and the Stokes force, $F_{S}=3 \pi \mu d_{C} v_{C}$, where $N$ is the number of nanoparticles in the complex agglomerate. Note that the buoyancy force is neglected since the density of the gas is much smaller than the density of the particle. From this we have $v_{C}=v_{p} N / k$, where $k=d_{C} / d_{p}$ is the ratio of complex-agglomerate size $d_{C}$ to primary particle size. The complex-agglomerate volume fraction $\phi_{C}$ would be given by $\phi_{C}=\left(N_{0} / N\right)(1 / 6) \pi d_{C}^{3}$, where $N_{0}$ is the number density of particles $\left[\phi=N_{0}(1 / 6) \pi d_{p}^{3}\right]$. Thus $\phi_{C}=\phi k^{3} / N$ and Eq. (2) can be rewritten as

$$
v_{g}=v_{p} k^{D-1}\left(1-k^{3-D} \phi\right)^{n},
$$

where the complex-agglomerate fractal dimension $D$ has been defined as $D=\ln N / \ln k$. The extension of the RZ equation for fluidized beds of nanoparticles was first proposed in Ref. [18] and has been used to estimate the complexagglomerate size in these systems (see Ref. [7] for a review).

The best fit of Eq. (3) to our data in the uniform fluidlike regime is plotted in Fig. 2 and gives $d_{C}=226 \mu \mathrm{m}( \pm 3 \mu \mathrm{m})$ and $D=2.59( \pm 0.001)$, which is in good agreement with previous measurements on the same nanopowder [7]. Experimental works reported in the literature for fluidization of different fluidized systems of fine and ultrafine particles show that $D$ is close to 2.5 , independently of particle size $[5,17,19]$. This finding is consistent with diffusion-limited aggregation (DLA) mechanism [20], in which self-similar 
fractal ramified patterns are grown via irreversible sticking of the aggregating units in a random motion. DLA seems to be the dominant mechanism of aggregation in fluidized beds of fine particles. In the case of nanoparticles, pre-existing simple agglomerates would agglomerate in the fluidized bed into complex agglomerates according to DLA mechanism $[18,19]$. Agglomerating units (simple agglomerates in the case of nanoparticles) are expected to display a diffusive dynamics in the fluidized bed, as inferred from diffusion experiments [21], and stick to agglomerates due to the prevailing of interparticle adhesion force on weight force. Thus complex agglomerates should appear as branched structures whose growth is controlled by a diffusive process and size is limited by flow shear.

\section{B. Effect of an alternating electric field}

In the absence of humidity the dominant interaction between nanoparticle agglomerates at contact arises from the van der Waals attractive force $F_{\mathrm{vdW}} \simeq A d_{a} / 24 z_{0}^{2}$ (it was shown in Ref. [22] that the van der Waals force prevailed over the electrostatic force arising from the natural charge of the agglomerates). Here $A$ is the Hamaker constant ( $A$ $\simeq 10^{-19} \mathrm{~J}$ ) and $z_{0} \simeq 3-4 \AA$ is the minimum intermolecular distance. In order to estimate the van der Waals force between the type of agglomerates shown in Fig. $3, d_{a}$ should be the typical size of their surface asperities [24]. Chaouki et al. [23] used $d_{a s}=0.2 \mu \mathrm{m}$ as typically used for the surface asperity size of micron-scale particles [24]. Taking $d_{a s}$ $\simeq 0.2 \mu \mathrm{m}$ we estimate $F_{\mathrm{vdW}} \sim 10 \mathrm{nN}$, which is susceptible to increase due to compaction [25]. When an alternating field $E=E_{0} \cos (\omega t)$ is applied, the naturally charged complex agglomerates are forced to oscillate in the stationary state at the same frequency of the field. The order of magnitude of complex-agglomerate charge, measured in a previous work by tracking complex-agglomerate trajectories under a dc electric field [22], is $Q=Q_{C} \sim 10^{-14}$ C. Since the electric force $Q_{C} E \sim 1 \mathrm{nN}$ is thus smaller than $F_{\mathrm{vdW}}$ it is likely that, at small oscillation frequencies, a solid powder layer remain deposited on the wall upon field reversal. This type of behavior may occur when the complex-agglomerate oscillation length $x_{C}$, defined as the horizontal distance traveled by the complex agglomerates during one oscillatory period due to the electric force, is comparable to the bed width $(\sim 1 \mathrm{~cm})$.

To have an estimation of $x_{C}$, long-range interactions will be disregarded and complex agglomerates will be considered as effective spheres. The drag force on a isolated sphere undergoing oscillations in a fluid with a velocity $v=v_{0} \cos (\omega t$ $+\varphi)$ is given by $F_{D}=a v+b d v / d t$, where $a=6 \pi \mu R(1+R / \delta)$, $b=3 \pi R^{2} \sqrt{2 \mu \rho / \omega}(1+2 R / 9 \delta), R$ is the sphere radius, $\mu$ is the fluid viscosity, $\rho$ is the fluid density, and $\delta=\sqrt{\mu / \rho \omega}$ is the penetration depth of the rotational fluid flow field around the sphere [26]. In the limit $\delta / R \rightarrow \infty$, as, for example, for a static electric field, $F_{D}$ converges to the Stokes drag force $F_{S}$. For nanoparticle complex agglomerates $\left(R=R_{C} \sim 100 \mu \mathrm{m}\right)$ fluidized by dry air, it is estimated $\delta \sim R$ for $f \sim 100 \mathrm{~Hz}$, which fits in the range of frequencies tested in our experiment, thus the Stokes equation is not applicable. Solving the equation of motion of a single sphere of mass $m$, the sphere oscillation

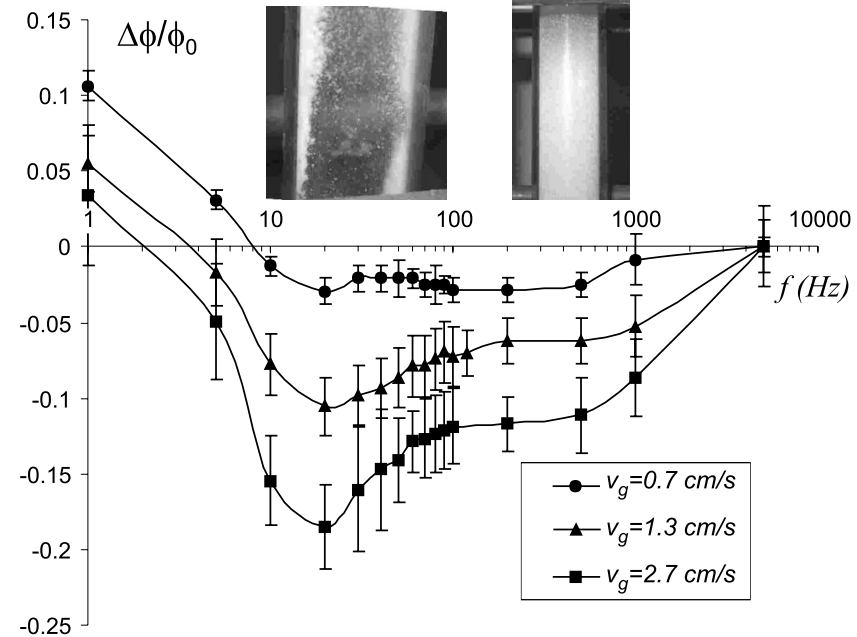

FIG. 4. Relative variation of the particle volume fraction vs electric field frequency. Root-mean-squared field is $1.25 \mathrm{kV} / \mathrm{cm}$. Photographs in the inset illustrate electrophoretic deposition (lefthand side, $v_{g}=2.7 \mathrm{~cm} / \mathrm{s}, f=1 \mathrm{~Hz}$ ) and enhanced expansion (righthand side, $v_{g}=2.7 \mathrm{~cm} / \mathrm{s}, f=20 \mathrm{~Hz}$ ).

length is $x=2 Q E_{0} / \omega \sqrt{a^{2}+\omega^{2}(m+b)^{2}}$. Using typical values in our experiment, it can be estimated that $x_{C} \sim 1 \mathrm{~cm}$ for $f$ of the order of hertzs.

On the other hand, in the limit of high frequency, the complex agglomerates will display very short oscillations. Since complex agglomerates are formed in an agglomerating process of pre-existing simple agglomerates, it is unlikely that this mechanism is affected by the electric field when $x_{C}$ is shorter that the size of smallest simple-agglomerate size $\left(d_{S} \sim 1 \mu \mathrm{m}[12]\right)$. Accordingly, and using typical values in our experiment, the field is expected to have no effect for $f$ above kilohertzs.

Figure 4 shows the relative variation of the particle volume fraction $\left(\Delta \phi / \phi_{0}\right)$ as a function of the oscillation frequency $f$ for fixed values of the root-mean-squared field $\left(E_{\mathrm{rms}}=1.25 \mathrm{kV} / \mathrm{cm}\right)$ and gas velocity. A square wave form has been used for practical convenience (the effect of waveform type is discussed below). It is seen that, in the limits of small and high frequency, the fluidization behavior depends on $f$ as it has been anticipated. For frequencies of the order of hertzs the main mechanism is electrophoretic deposition of the complex agglomerates onto the walls as seen in the case of a dc field, which gives rise to bed channeling and collapse (inset of Fig. 4). For frequencies of the order of kilohertzs and larger, the field does not play a significant role on bed expansion. In the range of intermediate frequencies bed expansion is enhanced as the inset of Fig. 4 illustrates. The rest of this paper is devoted to analyze this latter effect.

The effect of the field strength and wave-form type in the range of intermediate frequencies is shown in Fig. $5(f$ $=20 \mathrm{~Hz}$ ). The data, when plotted as a function of the rootmean-squared field $E_{\mathrm{rms}}$, adjust to a single trend independent of the wave-form type, indicating that enhanced bed expansion is the result of a time averaged process. Bed expansion is further enhanced as the strength of the field is increased at constant gas velocity, and for $E_{\mathrm{rms}}>1.5 \mathrm{kV} / \mathrm{cm}$ the mass of elutriated powder off the bed becomes appreciable. Since in 


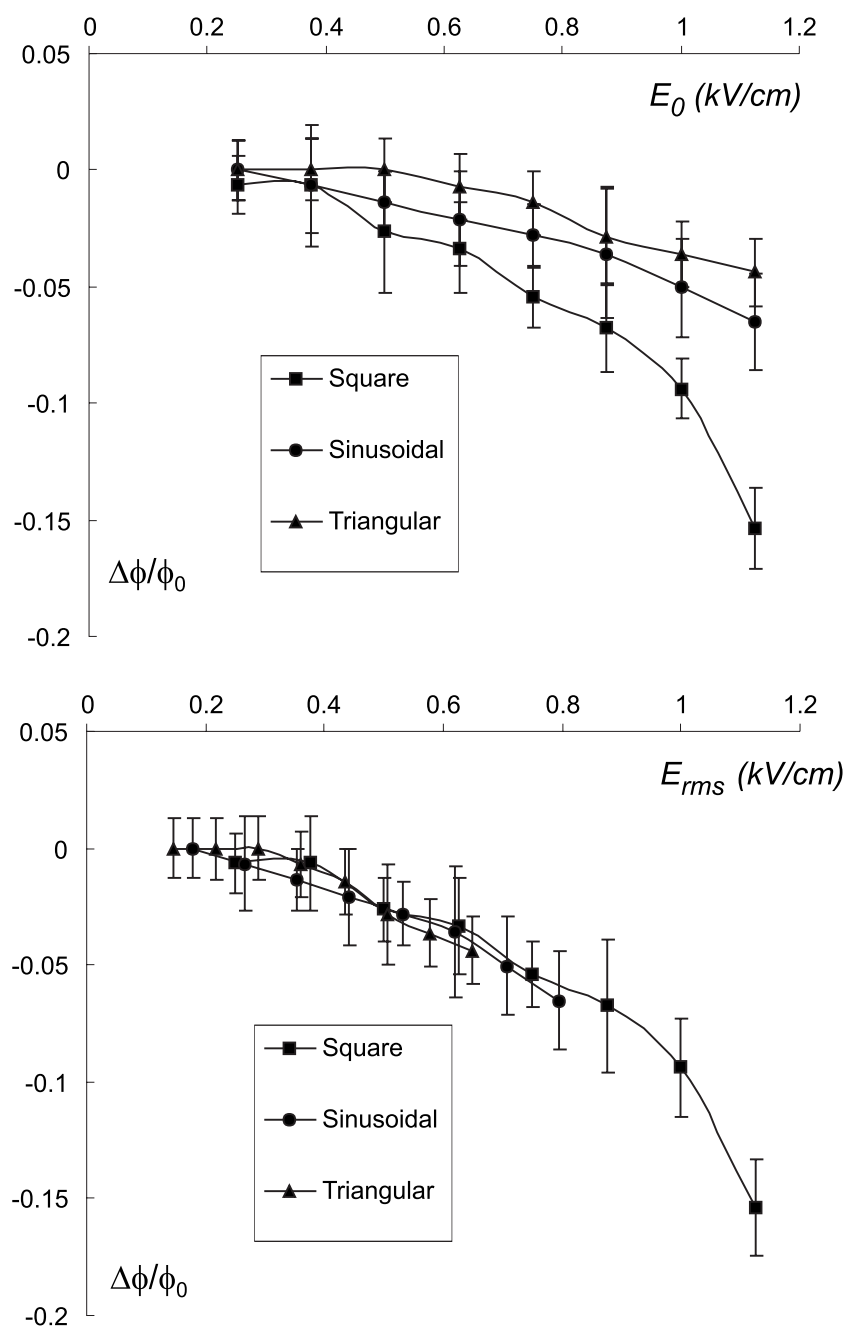

FIG. 5. Top: Relative variation of the particle volume fraction vs peak field strength $E_{0}$ for three different wave-form types. Bottom: The same data is shown as a function of the root-mean-squared field strength $E_{\mathrm{rms}}$. Field frequency and superficial gas velocity are fixed to $20 \mathrm{~Hz}$ and $v_{g}=2.7 \mathrm{~cm} / \mathrm{s}$, respectively.

a uniformly fluidized bed, the fluctuation velocity of the fluidized units (in our case complex agglomerates) is expected to be similar to gas velocity [27], it would be expected that the field effect becomes relevant for field strengths giving rise to horizontal velocities of the complex agglomerates comparable to gas velocity. From the above calculations, and using typical values in our experiment, the complexagglomerate root-mean-squared velocity $v_{\mathrm{rms}}$ is estimated to be similar to the gas velocity $\left(v_{\mathrm{rms}} \sim v_{g} \sim 1 \mathrm{~cm} / \mathrm{s}\right)$ for $E_{\mathrm{rms}}$ $=1 \mathrm{kV} / \mathrm{cm}$. On the other hand, for $E=0.2 \mathrm{kV} / \mathrm{cm}$, it is $v_{\mathrm{rms}}$ $\sim 1 \mathrm{~mm} / \mathrm{s}$, indicating that complex-agglomerate fluctuations overwhelm the complex-agglomerate forced oscillations for this field strength. Thus, no field effect should be observed for fields of this strength and smaller as seen experimentally.

\section{ANALYTICAL MODELING}

According to the mRZ equation [Eq. (3)], enhanced bed expansion by the electric field could be attributed to a varia- tion of the complex-agglomerate size. The strong dependence of $\Delta \phi / \phi_{0}$ on the root-mean-squared field strength suggests that there must be a relationship between the complexagglomerate size and $E_{\mathrm{rms}}$. In Ref. [25] a model was derived to estimate the size of agglomerates of micrometric particles in a fluidized bed subjected to the gravity field. We will carry out in this work a parallel derivation to estimate complex agglomerate size in the electrofluidized bed of nanoparticles, as effective particles undergoing agglomeration due to attractive forces. The novelty of the present analysis consists of extending it to nanoparticle agglomeration, including the effect of the electric field. In order to estimate theoretically the complex-agglomerate size in the electrofluidized bed of nanoparticles, simple agglomerates, which exist before fluidization, will be considered as effective particles undergoing agglomeration due to attractive forces between each other. This attractive force is counterbalanced by the gas flow shear to support the weight of the complex agglomerate in the gravity field plus the shear due to their forced oscillations in the electric field. The balance between the overall timeaveraged shear and the attractive force will determine the size of the complex agglomerates.

The vertical complex-agglomerate weight force $W_{C}$, which acts uniformly through the agglomerate body, is balanced by the hydrodynamic friction from the surrounding gas, which acts mainly at its surface due to the flow screening effect. From this balance, and using a spring model [25], the typical strain on the surface of the complex agglomerate can be estimated as $\gamma_{g} \sim W_{C} / K_{C} R_{C}$. Here $W_{C}=N_{S} W_{S}$, where $N_{S}$ is the number of simple agglomerates in the complex agglomerate and $W_{S}$ is the simple-agglomerate weight force. $K_{C}$ is the complex-agglomerate spring constant, which, using a theory on the elastic properties of random percolating systems [28], can be obtained from the simple-agglomerate spring constant $K_{S}$ and the elasticity exponent $\beta\left[K_{C}\right.$ $\sim K_{S} /\left(k_{C}\right)^{\beta}$, where $k_{C}$ is the ratio of complex-agglomerate radius $R_{C}$ to simple-agglomerate radius $R_{S}$ and $\beta=3$ in $3 \mathrm{D}$ ] [25]. The complex-agglomerate oscillations forced by the horizontal electric field give rise to an additional shear force to balance the electric force $Q_{C} E$. The root-mean-squared shear strain is thus increased up to $\gamma_{\mathrm{rms}}=\sqrt{\left(\gamma_{g}\right)^{2}+\left(\gamma_{E}\right)^{2}}$, where $\gamma_{E} \sim Q_{C} E_{\mathrm{rms}} / K_{C} R_{C}$. Thus, the shear force, which acts on the simple agglomerate attached to the outer layer of the complex agglomerate, is $F_{s} \sim K_{S} \gamma_{\mathrm{rms}} R_{S}$ $\sim\left(k_{C}\right)^{D+2} \sqrt{W_{S}^{2}+\left(Q_{S} E_{\mathrm{rms}}\right)^{2}}$. Here it is assumed $N_{S}=\left(k_{C}\right)^{D}$ and that the complex-agglomerate charge is equally distributed among the simple agglomerates $\left(Q_{C}=N_{S} Q_{S}\right.$, where $Q_{S}$ is the simple-agglomerate charge). Simple agglomerates are attached to the complex agglomerate as long as the shear force is smaller than the attractive force. Thus, the limit condition $F_{s}=F_{\mathrm{vdW}}$ leads to the equation

$$
k_{C} \sim \mathrm{Bo}^{1 /(D+2)}
$$

for estimating the complex-agglomerate size $d_{C}$, where the agglomerate Bond number Bo is defined as the ratio of $F_{\mathrm{vdW}}$ to $\sqrt{W_{S}^{2}+\left(Q_{S} E_{\mathrm{rms}}\right)^{2}}$. The simple-agglomerate weight is $W_{S}$ $=N_{p} W_{p}$, where $W_{p}$ is the nanoparticle weight and $N_{p}$ is the number of primary nanoparticles in the simple agglomerate, which is assumed to be $N_{p}=\left(d_{S} / d_{p}\right)^{D}$ [7]. Using a typical 


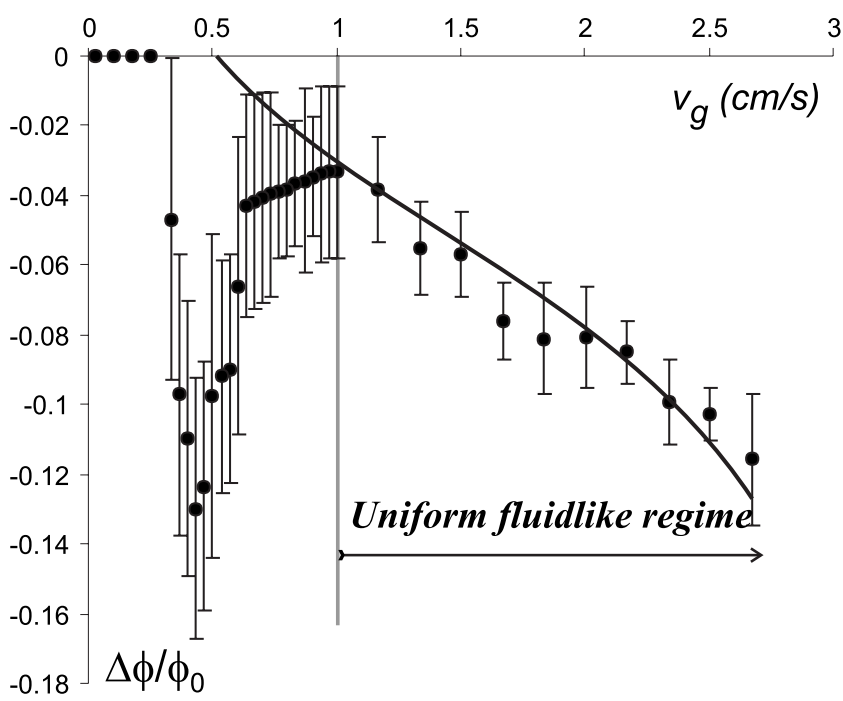

FIG. 6. Relative variation of the particle volume fraction vs the superficial gas velocity. Oscillation frequency and electric field strength are fixed to $500 \mathrm{~Hz}$ and $E_{\mathrm{rms}}=1.25 \mathrm{kV} / \mathrm{cm}$, respectively. The line is the model prediction.

value of $d_{S}=30 \mu \mathrm{m}$, as inferred from the work of Nam et al. [5], it is predicted that $d_{C}^{0}=k_{C}^{0} d_{S} \sim 150 \mu \mathrm{m}(E=0)$ for our silica nanoparticles, which is in accordance with experimental values reported in the literature from noninvasive imaging techniques [7] and with our previous estimation in this paper. When the electric field is turned on, the simple-agglomerate charge $Q_{S}$ is needed to estimate the complex-agglomerate size. Provided that simple agglomerates are unaltered by the electric field, it is $Q_{S}=Q_{C}^{0} / N_{S}^{0}$, where $Q_{C}^{0}$ and $N_{S}^{0}$ are the complex-agglomerate charge and number of simple agglomerates in the complex agglomerate for $E=0$, respectively. Thus

$$
k_{C}=k_{C}^{0}\left[1+\left(\frac{Q_{C}^{0} E_{\mathrm{rms}}}{\left(k_{C}^{0}\right)^{D}}\right)^{2}\right]^{-1 /[2(D+2)]} .
$$

Typically $\left(E_{\mathrm{rms}} \sim 1 \mathrm{kV} / \mathrm{cm}, Q_{C}^{0} \sim 10^{-14} \mathrm{C}\right)$, the relative decrease of $d_{C}$ and $Q_{C}$ are about $15 \%$ and $30 \%$, respectively. The effect of the field on bed expansion can be taken into account by using the complex-agglomerate predicted size [Eq. (5)] in the mRZ equation [Eq. (3)]. Figure 6 shows $\Delta \phi / \phi_{0}$ as a function of the gas velocity for $E_{\mathrm{rms}}$ $=1.25 \mathrm{kV} / \mathrm{cm}$. The model prediction that fits better to the data in the fluidlike regime is plotted. Complex-agglomerate size, in the absence of electric field, and $D$ are taken from our previous bed expansion measurements $\left(d_{C}=226 \mu \mathrm{m}, D\right.$ $=2.59)$. Remarkably, the prediction fits well to the data for a value of the complex-agglomerate charge $\left(Q_{C}^{0}=1.9\right.$ $\times 10^{-14} \mathrm{C}$ ) similar to the value directly measured elsewhere [22]. Moreover, this value gives a charge to mass ratio in the range $10^{-5}-10^{-4} \mathrm{C} / \mathrm{kg}$, which is comparable to the values indirectly measured by Tardos et al. [2] from the electrical current in a Faraday cage induced by coarse grains in dry fluidized beds. Interestingly, for this charge value, the electric force on the complex agglomerate is approximately equal to the complex-agglomerate weight $\left(Q_{C}^{0} E \simeq W_{C}^{0}\right.$ $\simeq 2 \mathrm{nN}$ ).

It is remarkable that the theoretical results fit reasonably well to experimental results for values of the agglomerate charge close to the experimentally measured values, which upholds the applicability of the analysis presented in Ref. [25], and extended in this paper, to predict agglomerate size from fundamental properties such as interparticle attractive force, particle size and density, and external fields. Due to the emerging applications of fluidization of nanoparticles, it is very important for the engineering community to have a simple tool to estimate beforehand agglomerate size, type of fluidization expected, fluidized bed expansion curve and packing in the solid state from fundamental physical parameters [7]. Since silica nanoparticles fluidize well without external aids, the effect of the electric field on bed expansion is not pronounced in the range of field strengths applied (less than 20\%). Note, however, that higher fields give rise to elutriation of the powder, which can be of interest for powder applications involving powder transport. It is also expected that an alternating field will have a relevant effect on the bubbling behavior exhibited by other nanoparticle systems such as titania [11]. In that case it is expected that the alternating field contributes to destabilize bubbles and homogenize fluidization. Experiments to corroborate this expectation are in progress.

In the transitional regime, from the fluidlike to solidlike behavior $\left(v_{g} \lesssim 1 \mathrm{~cm} / \mathrm{s}\right)$, it is expected that the main physical mechanism by which the field affects fluidization is destabilization of channels that allowed gas to bypass the bed, thus favoring bed expansion. At the jamming transition $\left(v_{g}\right.$ $\simeq 0.5 \mathrm{~cm} / \mathrm{s}$ ), the weight of the agglomerates is just sustained by the gas flow. For slightly smaller gas velocities, the attractive van der Waals force between agglomerates holds them in a weak solid network that spans through the bed. The attractive van der Waals force between agglomerates $\left(F_{\mathrm{vdW}}\right.$ $\sim 10 \mathrm{nN}$ ) is just slightly larger than the electric force, thus gas flow perturbations, helped by the electric field, may still drive the bed into the fluidlike regime locally in the close vicinity of the jamming transition, which leads to enhanced bed expansion effect. The effect of the field is definitely neutralized for gas velocities below $v_{g} \simeq 0.3 \mathrm{~cm} / \mathrm{s}$.

\section{CONCLUSION}

The behavior of a cross-flow-type electrofluidized bed of nanoparticles has been studied. It has been found that bed expansion is enhanced for electric field strengths of about $1 \mathrm{kV} / \mathrm{cm}$ and oscillation frequencies of tens to hundreds of hertzs. For oscillation frequencies of the order of hertzs, or smaller, bed expansion is hindered due to electrophoretic deposition of the agglomerates onto the vessel walls, whereas for oscillation frequencies of the order of kilohertzs, or larger, electrophoresis is nullified and bed expansion is not affected. According to a proposed model, the size of nanoparticle agglomerates stems from the balance between shear, which depends on field strength, and van der Waals forces. The optimum field strength for enhancing bed expansion produces an electric force on the agglomerates similar to 
their weight, while the oscillation velocity of the agglomerates is similar to the gas velocity. Higher field strengths give rise to excessive elutriation, while smaller ones do not affect appreciably bed expansion. The electric field is screened by the van der Waals attractive interaction when the superficial gas velocity is decreased below $v_{g} \simeq 0.3 \mathrm{~cm} / \mathrm{s}$ and the fluidized bed of nanoparticles is jammed. It is foreseen that because of their huge specific surface area and enhanced uniform fluidization, electrofluidized bed of nanoparticles could improve the efficiency of fluidized beds based devices such as gas cleaning filters.

\section{ACKNOWLEDGMENTS}

We acknowledge Spanish Government (Contract No. FIS2006-03645) and Junta de Andalucia (Contract No. FQM 421).
[1] J R. Melcher, Proc. IEEE 65, 1659 (1977).

[2] G. Tardos et al., Ind. Eng. Chem. Fundam. 22, 445 (1983).

[3] R. Elsdon and C. J. Shearer, Chem. Eng. Sci. 32, 1147 (1977).

[4] D. Geldart, Powder Technol. 7, 285 (1973).

[5] C. Nam, R. Pfeffer, R. N. Dave, and S. Sundaresan, AIChE J. 50, 1776 (2004).

[6] J. M. Valverde and A. Castellanos, Phys. Rev. E 75, 031306 (2007).

[7] J. M. Valverde and A. Castellanos, Chem. Eng. Sci. 62, 6947 (2007).

[8] S. Matsuda, H. Hatano, and A. Tsutsumi, Chem. Eng. J. 82, 183 (2001).

[9] Z. Hao et al., Powder Technol. 183, 46 (2008).

[10] V. Jiradilok et al., Powder Technol. 164, 33 (2006).

[11] J. M. Valverde et al., AIChE J. 54, 86 (2008).

[12] W. Yao, G. Guangsheng, W. Fei, and J. Wu, Powder Technol. 124, 152 (2002).

[13] P. Wiltzius, Phys. Rev. Lett. 58, 710 (1987).

[14] J. F. Richardson and W. N. Zaki, Trans. Inst. Chem. Eng. 32, 35 (1954).

[15] G. K. Batchelor, J. Fluid Mech. 119, 379 (1982); G. K. Batchelor and C. S. Wen, ibid. 124, 495 (1982).

[16] J. M. Valverde et al., Europhys. Lett. 54, 329 (2001).
[17] A. Castellanos, J. M. Valverde, and M. A. S. Quintanilla, Phys. Rev. E 64, 041304 (2001).

[18] J. M. Valverde and A. Castellanos, AIChE J. 52, 838 (2006).

[19] X. S. Wang, V. Palero, J. Soria, and M. J. Rhodes, Chem. Eng. Sci. 61, 8040 (2006).

[20] T. A. Witten and L. M. Sander, Phys. Rev. Lett. 47, 1400 (1981).

[21] J. M. Valverde, A. Castellanos, and M. A. S. Quintanilla, Phys. Rev. Lett. 86, 3020 (2001).

[22] J. M. Valverde, M. A. S. Quintanilla, M. J. Espin, and A. Castellanos, Phys. Rev. E 77, 031301 (2008).

[23] J. Chaouki, C. Chavarie, D. Klvana, and G. Pajonk, Powder Technol. 43, 117 (1985).

[24] K. Rietema, The Dynamics of Fine Powders (Elsevier, London, 1991).

[25] A. Castellanos, J. M. Valverde, and M. A. S. Quintanilla, Phys. Rev. Lett. 94, 075501 (2005).

[26] L. D. Landau and E. M. Lifshitz, Fluid Mechanics, Course of Theoretical Physics Vol. 6 (Pergamon, New York, 1995).

[27] P. N. Segre, F. Liu, P. Umbanhowar, and D. A. Weitz, Nature (London) 409, 594 (2001); P. N. Segre, E. Herbolzheimer, and P. M. Chaikin, Phys. Rev. Lett. 79, 2574 (1997).

[28] Y. Kantor and I. Webman, Phys. Rev. Lett. 52, 1891 (1984). 